\title{
Probabilistic Broadcast for Flooding in Wireless Mobile Ad hoc Networks
}

\author{
Yoav Sasson David Cavin André Schiper \\ \{yoav.sasson, david.cavin, andre.schiper\} @epfl.ch \\ École Polytechnique Fédérale de Lausanne (EPFL) \\ 1015 Lausanne, Switzerland
}

\begin{abstract}
Although far from optimal, flooding is an indispensable message dissemination technique for network-wide broadcast within mobile ad hoc networks (MANETs). As such, the plain flooding algorithm provokes a high number of unnecessary packet rebroadcasts, causing contention, packet collisions and ultimately wasting precious limited bandwidth. We explore the phase transition phenomenon observed in percolation theory and random graphs as a basis for defining probabilistic flooding algorithms.

By considering ideal and realistic models, we acquire a better understanding of the factors that determine phase transition, the consequences of the passage to realistic MANET conditions and to what extent we may benefit from probabilistic flooding in real MANET networks.
\end{abstract}

\section{INTRODUCTION}

Mobile ad hoc networks (MANETs) are self-organizing mobile wireless networks that do not rely on a preexisting infrastructure to communicate. Nodes of such networks have limited transmission range, and packets may need to traverse multiple other nodes before reaching their destination. Research in MANETs was initiated 20 years ago by DARPA for packet radio projects [13], but has regained popularity nowadays due to the widespread availability of portable wireless devices such as cell phones, PDAs and WiFi / Bluetooth enabled laptops.

Because of the ever-changing topology of MANETs, broadcasting [19] is a fundamental communication primitive, essential to ad hoc routing algorithms (e.g., [20], [5]) for route discovery. The usual approach for broadcasting is through flooding. Flooding is well suited for MANETs as it requires no topological knowledge. It consists in each node rebroadcasting a message to its neighbors upon receiving it for the first time.

Although straightforward, flooding is far from optimal and generates a high number of redundant messages, wasting valuable limited resources such as bandwidth and energy supplies. Besides research mentioned in Section II, more effort has been devoted to defining MAC and routing algorithms adapted to MANETs, than to flooding. Since flooding is a

The work presented in this paper was supported by the National Competence Center in Research on Mobile Information and Communication Systems (NCCR-MICS), a center supported by the Swiss National Science Foundation under grant 5005-67322. low-level primitive, optimizing it will drastically improve the overall performance of MANETs.

One direction to optimize flooding is to take a probabilistic approach. In order to flood, a node in the network broadcasts a message with probability $p$ and takes no action with probability $1-p$. In our paper we explore the possibility of applying a phenomenon well studied in percolation theory and random graphs, phase transition, as a basis for selecting $p$. Above a certain threshold for $p$, in graphs of a certain size for random graphs and lattices of a certain density for percolation, an infinite spanning cluster abruptly appears instead of a set of finite clusters. An infinite spanning cluster is a unbounded connected component, which if transposed to a MANET would translate in the very high probability of the existence of a multi-hop path between any two nodes within the network.

To the best of our knowledge, besides [12], previous publications having studied probabilistic broadcast for flooding in MANETs [6], [16] have not done so within the context of phase transition. This paper contributes in a first stage to a better understanding of the various factors that influence phase transition in ideal MANET environments (no packet collisions). By opposition to traditional theoretical phase transition analysis and simulation, we specifically consider factors that would typically intervene within probabilistic algorithms deployed on MANETs. In a second stage, we illustrate the consequences of considering realistic effects such as packet collisions and node mobility. To the contrary of [12], we concentrate on pure flooding in order to understand the variations in performance due solely to the parameters simulating realistic MANET environments. Our results therefore provide a general understanding of the behavior to be expected from probabilistic flooding.

The remainder of the paper is organized as follows. Section II gives an overview of other works that seek to reduce the overhead of flooding in MANETs. In Section III we introduce the phase transition phenomenon, known results, and how it may benefit flooding in MANETs. In Section IV we present two models for which we study the phase transition behavior. Section V contains simulations and results of our algorithms. Finally, we conclude and describe future work in Section VI. 


\section{RELATED WORK}

In this section we examine related work which directly or indirectly aims at reducing the number of broadcast packets generated by the flooding algorithm.

The high number of redundant broadcast packets due to flooding in MANETs has been referred to as the Broadcast Storm Problem in [16]. The paper proposes several schemes, of which one probabilistic, in order to reduce the number of broadcast packets while maintaining high reliability. More recently, [6] provides a comparative study of broadcasting algorithms, including probability based methods. Given the scenarios and results in these two papers, it is difficult to make any statement regarding phase transition (Section III).

As for deterministic studies of the problem, [18] and [15] make use of local topology knowledge in order to avoid unnecessary rebroadcasts by comparing the added coverage between the rebroadcast of a destination node to that of the source node at each hop. [18] enhances the algorithm by taking into consideration statistical information about broadcast duplicates, whereas [15] enhances the algorithm by taking into account two-hop coverage. [21] restricts flooding to a subset of nodes ("multipoint relays") by selecting for each node a minimum number of one-hop neighbors covering all second-hop neighbors. [17] ${ }^{1}$ and more recently [23] (with an optimized approach) explore the idea of superimposing a communications graph - a cluster - over the network so that only particular nodes rebroadcast the packets. Albeit reducing the number of rebroadcast packets, constructing and maintaining the clusters introduce a new source of overhead in a mobile network.

Other fields such as percolation theory and random graphs have recently been a source of inspiration for designing solutions within MANETs. Both are based on a probabilistic model and exhibit an interesting phenomenon called phase transition. They will be presented in more detail in Section III.

Phase transition has been applied to reduce traffic for multicast in wired networks [2], to study optimum power ranges for connectivity [3], [11] and for enhancing connectivity in hybrid MANET/Wired networks [7]. Only recently however, and in parallel with our research, have characteristics from these fields been applied to reduce flooding in MANETs:

- [14] points out that the phase transition phenomenon also occurs in MANETs and may be taken advantage for the elaboration of probabilistic algorithms such as flooding and routing within such networks.

- [12] studies a gossip-based approach to flooding. Through simulations the authors show that for large networks, a simple gossiping uses up to $35 \%$ fewer messages than flooding, and that the performance of AODV routing [20] relying on gossip-based flooding is improved even in small networks of 150 nodes.

This paper is based on the same inspiration as [14] and [12], yet we obtain different results and gain a better understanding of the phase transition behavior.

\footnotetext{
${ }^{1}$ The goal of this paper is primarily to provide reliable broadcast delivery.
}

\section{The Phase Transition Phenomenon}

A phase transition is a phenomenon where a system undergoes a sudden change of state: small changes of a given parameter in the system induces a great shift in the system's global behavior. This abrupt transition occurs at a specific value $p_{c}$ called the critical point or critical threshold. Below $p_{c}$ the system is said to be in a subcritical phase - the global behavior is non-existent. Above $p_{c}$ the system is in a supercritical phase and the global property may be almost surely observed. Figure 1 illustrates the phase transition probability $\theta$ given the probability $p$ of a problem specific parameter $\lambda$. $L$ denotes the size of the system considered.

It would be extremely cost-efficient to observe phase transition in a probabilistic flooding algorithm within all or known subsets of MANET topologies. The implication within such cases would be that there exists a certain probability threshold $p_{c}<1$ at which the flooded message will almost surely reach all nodes within multihop broadcast reach. Broadcasting with a probability $p>p_{c}$ will not provide any significant improvement. We now present two areas of research where phase transition applies in order to extract models for MANETS in Section IV and study their phase transition properties.

\section{A. Percolation Theory}

Percolation theory studies the flow of fluid in random media and has been generally credited as being introduced in 1957 by Broadbent and Hammersley [4]. Two main twodimensional lattice square percolation models are studied, site percolation and bond percolation. In the bond percolation model (Figure 2(a)), each edge of the lattice is said to be open with probability $p$ and closed with probability $1-p$. The fluid flows through the open edges of the lattice. The site percolation model on the other hand considers the lattice squares or sites to be the relevant entities (Figure 2(b)): A lattice site is open with probability $p$ and closed with probability $1-p$, and the fluid flows from open site to open site across the lattice. Figure 2(b) illustrates an example of site percolation with $p \simeq 0.55$.

Phase transition in percolation models is observed as the change of state between having a finite number of clusters and having one infinite cluster. A cluster is a set of connected entities (edges for bond percolation and sites for site percolation). A cluster that reaches from one side of the lattice to the other is said to be an infinite cluster. Percolation theory

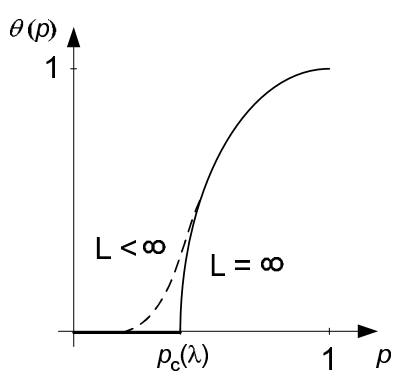

Fig. 1. Phase Transition 
studies the existence and value $p_{c}$ for which phase transition occurs, as well as cluster numbers, sizes and structures.

Percolation theory has numerous interesting applications to cases which involve some form of propagation or connectivity such as the spreading of infectious diseases with regard to population density or the spreading of forest fires. The question is whether results may also be derived for message propagation within real-world MANETs.

Great effort within percolation theory has been devoted to finding the exact value of $p_{c}$ at which the phase transition occurs. Unfortunately, $p_{c}$ is not universal but specific to each lattice geometry. Besides a few distinct cases, there is no general analytical formula to obtain $p_{c}$, which is usually computed case by case through Monte Carlo simulations.

\section{B. Random Graphs}

Another predominant area of research for phase transition is Random Graphs. A random graph $G$ is a graph where the number of nodes, edges and connections between them are determined in some random manner. The phase transition property has been well studied in the context of random graphs. Erdős and Rényi [8] have shown that the probability of a random graph being connected tends to 1 if the number of edges $E$ is greater than $p_{c}(E)=\frac{N \log N}{2}$. Although the results of Erdôs and Rényi are for large values of $N$, Frank and Martel have shown by simulation in [9] that phase transition occurs also in graphs of moderate size (between 30 to 480 vertices). In other words, we may view $p_{c}(E)$ as a critical value for the number of edges above which a phase transition will occur, resulting in a quick convergence for obtaining a connected graph. As such, we are not able to use random graphs to represent MANETs: In random graphs, an edge may connect any two vertices's in the euclidean plane. In MANETs however, communication links connect nodes that are within communication range only. In Section IV-B we describe the Fixed Radius Model which is an ideal representation of MANET topologies. It remains a question whether results as in [8], [9] may be observed in the fixed radius model.

\section{Discussion}

Phase transition properties depend greatly on the graph geometry. There is no general theoretical result that enables

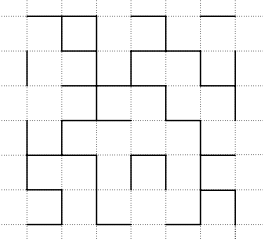

(a) Bond Percolation (open edges are in bold)

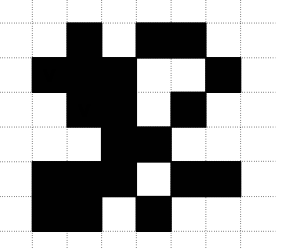

(b) Site Percolation (open sites are in black)
Fig. 2. Bond and Site Percolation us to determine the critical threshold $p_{c}$ at which the transition will take place, if at all. Therefore $p_{c}$ will have to somehow be approximated. Furthermore, the few results we have from percolation theory are for infinite lattices $(L=\infty)$. As we take smaller configurations $(L \ll \infty)$, the transition from the subcritical to the supercritical state becomes less abrupt. The dashed tail of the graph in Figure 1 illustrates that in non infinite lattice configurations, the probability of percolation taking place becomes linear. We must therefore determine graph characteristics for which border effects are not significant.

\section{Applying Phase Transition to Flooding}

Similarly to wired networks, we may model a MANET by a graph. Let $G=(V, E)$ be an undirected graph. A vertex $v_{i} \in V$ represents a mobile node, and an edge $e_{i j} \in E$ means that the nodes $i$ and $j$ are within communication range of each other. Within this paper we assume that all mobile nodes possess the same constant transmission range, and do not consider other properties such as energy levels or consumption.

Given a broadcast source node $S$, let $G_{B}$ be the connected subgraph of $G$ representing all nodes that will receive the broadcast message by flooding $\left(S \in G_{B}\right)$ (Figure 3). Since the message reaches all the nodes in the graph, $G_{B}$ may be thought of as an infinite open cluster as defined in Section III-A. An efficient probabilistic algorithm will remove edges from $G_{B}$ while still remaining above $G_{B}$ 's percolation threshold $p_{c}$, thus maintaining the infinite open cluster. By remaining in the supercritical phase, we expect to observe a significant reduction of message traffic due to flooding while minimizing the loss of reachability.

We must however ultimately take into consideration that real-world MANETs differ from mathematical graphs on several points. The differences that impact phase transition properties are:

1) Typical real-world MANETs as we see them are not infinite but may be composed of a few tens to a few thousand nodes. Border effects may therefore eventually impact the system's behavior.

2) Nodes may join or leave the network for various reasons, constantly modifying the network's density over time. This directly affects the network's phase transition properties.

3) Packet loss: Packets within a MANET are lost due to packet collisions and contention as well as node

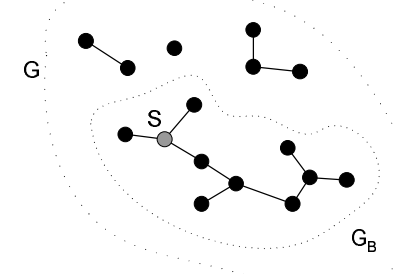

Fig. 3. $G_{B}$ Subgraph 
rithms in which nodes would dynamically adjust the probability $p$ for probabilistic flooding based on local graph topology information. In our paper we have made the assumption that all nodes possess the same transmission range. Another potential area for study would be to understand within probabilistic flooding the combined effects on MANETs performance of modifying the nodes' transmission range $r$ with regard to $p$.

\section{ACKNOWLEDGMENT}

We would like to thank Patrick Thiran for our discussions on percolation theory that have led to this paper.

\section{REFERENCES}

[1] The network simulator-ns-2. http://http://www.isi.edu/nsnam/ns.

[2] Kenneth P. Birman, Mark Hayden, Oznur Ozkasap, Zhen Xiao, Mihai Budiu, and Yaron Minsky. Bimodal multicast. ACM Transactions on Computer Systems, 17(2):41-88, 1999.

[3] Lorna Booth, Jehoshua Bruck, Massimo Franceschetti, and Ronald Meester. Covering algorithms, continuum percolation and the geometry of wireless networks.

[4] S.R. Broadbent and J.M Hammersley. Percolation processes I. crystals and mazes. In Proceedings of the Cambridge Philosophical Society, volume 53, pages 629-641, 1957.

[5] J. Broch, D.B. Johnson, and D.A. Maltz. The dynamic source routing protocol for mobile ad hoc networks (DSR). Internet draft (draft-ietfmanet-dsr-07.txt), Feb 2002. Work in Progress.

[6] T. Camp and B. Williams. Comparison of broadcasting techniques for mobile ad hoc networks. In Proceedings of The Third ACM International Symposium on Mobile Ad Hoc Networking and Computing (MOBIHOC 2002), Lausanne, Switzerland, Jun 2002.

[7] O. Dousse, P. Thiran, and Martin Hasler. Connectivity in ad-hoc and hybrid networks. In Proceedings of IEEE Infocom 2002, New York, June 2002.

[8] P. Erdős and A. Rényi. On the evolution of random graphs. In Publications of the Mathematical Institute of the Hungarian Academy of Sciences, volume 5, pages 17-61, 1960.

[9] J. Frank and C.U. Martel. Phase transitions in the properties of random graphs. In Principles and Practice of Constraint Programming (CP-95), Cassis, France, 1995.

[10] IEEE Working Group. Wireless LAN medium access control (MAC) and physical layer (PHY) specifications.
IEEE specification (http://standards.ieee.org/getieee802/download/802.11b1999.pdf), Sep 1999. Work in Progress.

[11] Piyush Gupta and P. R. Kumar. Critical power for asymptotic connectivity in wireless networks. In W. M. McEneany, G. Yin, and Q. Zhang, editors, Stochastic Analysis, Control, Optimization and Applications: A Volume in Honor of W.H. Fleming, pages 547-566. Birkhauser, Boston, 1998.

[12] Zygmunt J. Haas, Joseph Y. Halpern, and Li Li. Gossip-based ad hoc routing. In IEEE INFOCOM, Jun 2002.

[13] John Jubin and Janet D. Tornow. The DARPA packet radio network protocol. 75(1):21-32, Jan 1987

[14] B. Krishnamachari, S.B. Wicker, and R. Béjar. Phase transition phenomena in wireless ad-hoc networks. In Proceedings of the Symposium on Ad-Hoc Wireless Networks (GlobeCom2001), San Antonio, Texas, Nov 2001.

[15] H. Lim and C. Kim. Multicast tree construction and flooding in wireless ad hoc networks. In 3rd ACM International Workshop on Modeling, Analysis and Simulation of Wireless and Mobile Systems, 2000.

[16] Sze-Yao Ni, Yu-Chee Tseng, Yuh-Shyan Chen, and Jang-Ping Sheu. The broadcast storm problem in a mobile ad hoc network. In Proceedings of the Fifth Annual ACM/IEEE International Conference on Mobile Computing and Networking, pages 151-162, Aug 1999.

[17] E. Pagani and G.P Rossi. Providing reliable and fault tolerant broadcast delivery in mobile ad-hoc networks. In ACM/Baltzer Journal of Mobile Networks and Applications (MONET), volume 4, pages 175-192, 1999.

[18] Wei Peng and Xi-Cheng Lu. On the reduction of broadcast redundancy in mobile ad hoc networks. In First Annual Workshop on Mobile Ad Hoc Networking \& Computing, pages 129-130, Aug 2000.

[19] Charles E. Perkins, Elizabeth M. Royer, and Samir R. Das. IP flooding in ad hoc networks. Internet draft (draft-ietf-manet-bcast-00.txt), Nov 2001. Work in Progress.

[20] Charles E. Perkins, Elizabeth M. Royer, and Samir R. Das. Ad hoc on-demand distance vector routing (AODV). Internet draft (draft-ietfmanet-aodv-10.txt), Jan 2002. Work in Progress.

[21] A. Qayyum, L. Viennot, and A. Laouiti. Multipoint relaying for flooding broadcast messages in mobile wireless networks. In Proceedings of the 35th Annual Hawaii International Conference on System Sciences (HICSS'02), Big Island, Hawaii, Jan 2002.

[22] Dietrich Stauffer and Amnon Aharony. Introduction to Percolation Theory. Taylor \& Francis, second edition, 1992.

[23] I. Stojmenovic, M. Seddigh, and J. Zunic. Internal nodes based broadcasting in wireless networks-. In Proceedings of the 34th Hawaii International Conference on System Sciences (HICSS 2001), Maui, Hawaii, Jan 2001. 